\title{
Emergence of Abstaining in Glauber Opinion Dynamics
}

\author{
Baolong Niu \\ LMIB and School of Mathematics and Systems Science \\ Beihang University \\ Beijing, China \\ niucentury@smss.buaa.edu.cn
}

\begin{abstract}
We study the evolution of Glauber opinion dynamics with abstaining and tunable threshold on random graphs. The phase diagram shows plentiful features in the space of the two parameters of the model, the threshold and the abstaining probability. It is found that the threshold that limits the agents to be stable plays an important role in the emerging of abstaining in a wide spread. And it can be obtained that the observables stay the same in frozen state whatever the initial density of 0 is. We also use the mean field calculations to verify the fact of linearity between the density of 0 and the abstaining probability.
\end{abstract}

Keywords-opinion dynamics; abstaining; mean field method; phase transition

\section{INTRODUCTION}

Opinion dynamics is an effective way to study the complicated social systems and relative phenomena. The goal is to describe how people affect each other, and possibly to predict where the common opinion goes [1]. Many models have been devised, and each is based on known models or involves a new type of dynamics. The main ingredients of a model are a given graph topology, representing the social network of interactions between individuals, and the state space, in which the states stand for the opinions people hold. A dynamics should be defined, that is a rule indicating how an agent and its neighbors affect each other. Also the main ingredients are the pattern and condition of the evolution, meaning the process of effecting and changing. The pattern concerns the changing ways, such as spin flip and abstaining referring to states of agents, and rewiring [2] referring to network topology. The condition concerns the activation of changing, which is often used as deterministic ones, probabilistic ones [3], or threshold [4].

While various ingredients lead to different models, the dynamics is the major difference between them. Among the last 50 years, many classical dynamics models were raised. For example, the Voter model (1973) [5,6,7] which considers the agent to be affected randomly by one of its

\author{
Wei Wei \\ LMIB and School of Mathematics and Systems Science \\ Beihang University \\ Beijing, China \\ weiw@buaa.edu.cn
}

neighbors, the Glauber model (1963) [8], which considers the agent to be affected by all of its neighbors, the Majority Rule model (1982) [9], which considers a randomly chosen group affects its agents, the Sznajd model (2000) [10,11], which considers a pair affect all its neighbors, etc.

Existing models seldom take the state 0 into account, which is the neutralization of +1 and -1 that are of support and opposition. We generally call it abstaining, which widely exists in social events. Our model is based on Glauber dynamics, and selects threshold condition. What we are to seek is the emerging of state 0 , according to the threshold $s$, and the abstaining probability $\varphi$. And we try to explain as some disciplines, which help to get expected results in social events.

\section{MODEL DESCRIPTION}

Our model starts from a random graph in the manner of Erdös-Rényi [12, 13] with $N$ nodes, and wiring probability $p=<k>/ N, \quad k \quad$ being the average degree of the graph. So, the network has a Poisson degree distribution. Agents are endowed with state 0 for probability $\rho_{0}{ }^{o}$, and for equal probability of spare. The dynamics of the model is defined by the following update rule:

(1) A node is selected at random, and if its state is +1 or -1 , follow the rule (2); otherwise, follow the rule (3).

(2) We indicate with $k$ the number of its neighbors and with $n_{c}$ the number of neighbors in the opposite state, and with $n_{h}$ the number of neighbors in the same state; If $\left(n_{h^{-}}\right.$ $\left.n_{c}\right) / k \geq s$, where $-1 \leq s \leq 1$, the node is stable and nothing happens. Otherwise, with probability $\varphi$, it changes its state to 0 (abstaining); and with probability $1-\varphi$, it changes its state to the opposite (spin flip).

(3) We now indicate with $n_{+}$the number of neighbors

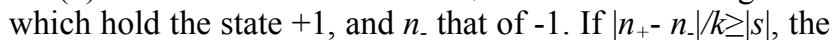
node changes its state to the majority's state between its neighbors (intervention). Otherwise, with probability $\varphi$, it stays abstaining; and with probability $1-\varphi$, it takes intervention. 

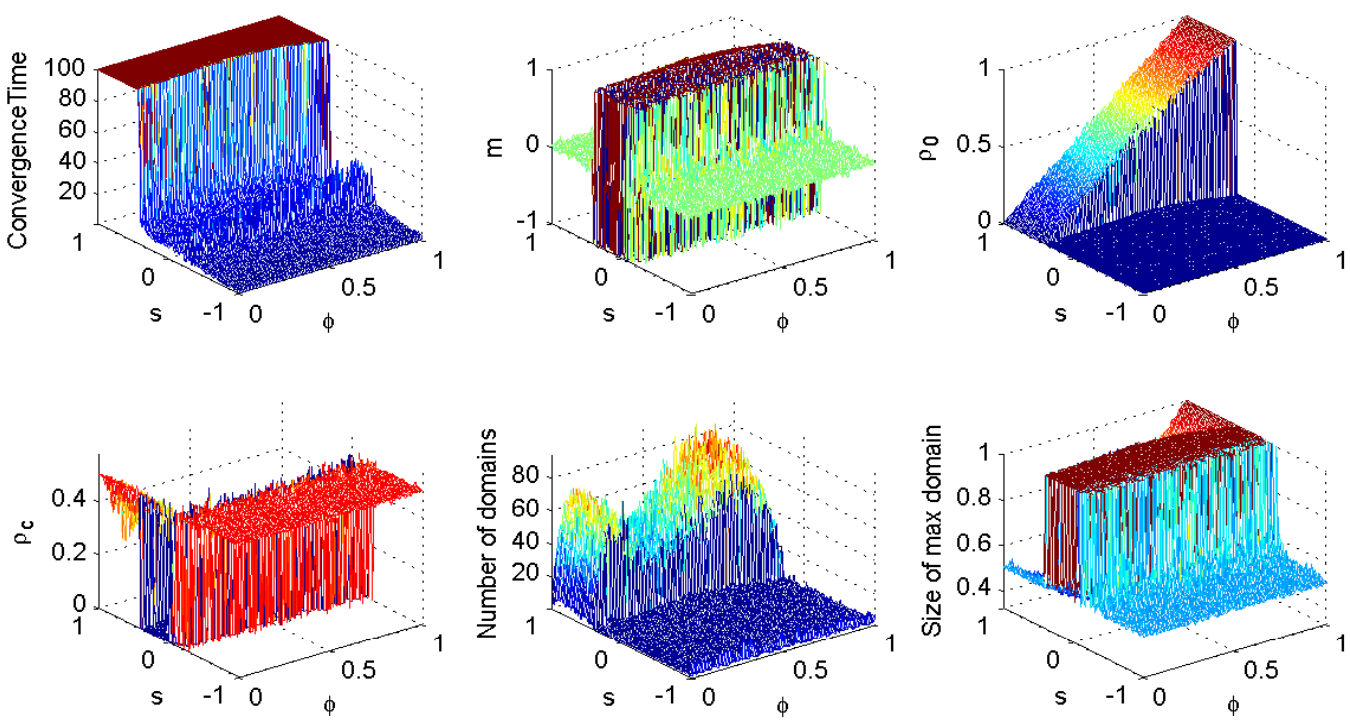

Figure 1. Values of variables depending on the threshold $s$, and the abstaining probability $\varphi$ : the convergence time (top left), the magnetization (top middle), the density of 0 (top right), the density of unsatisfied links (bottom left), the number of homogeneous domains (bottom middle), and the size of the largest domain (bottom right). Data are obtained from numerical simulations on a graph with $N=10000,<k>=10$, and the initial density of $0 \rho_{0}{ }^{\circ}=0$.

The model has two parameters: the threshold $s$, and the abstaining probability $\varphi$. The threshold sets the minimum difference between the number of the neighbors in the same state and that in the opposite state to be stable that the node must have. And the abstaining probability shows the sensitivity of people getting involved in the social events, or the desire of people to take a definite attitude to an event. We introduce the rule (3) to accord with the rule (2), they jointly express that $s$ is the limit to be stable, and $\varphi$ is the tendency to abstaining.

To study the features of the phase diagram of the model, we monitor the behavior of some observables. They are the convergence time $t_{c}$, the magnetization $m$, the density of nodes with state $0 \rho_{0}$, the density of unsatisfied links $\rho_{c}$, the number of homogeneous domains, and the relative size of the largest domain. Here we define the convergence time as the time needed to reach a frozen global state. We count it as units obtained through dividing number of steps that the experiment takes to get frozen, by the total number of the nodes. And we settle the terminal condition of an experiment as following: the evolution ends when either the graph has been unchanged for 1 unit, or the time has been 100 units. The magnetization $m$ comes from the statistical physics, which means the average value of all states. If we note $\rho_{+}$to be density of nodes with state +1 , and $\rho_{-}$to be density of nodes with state -1 . Then the magnetization $m=\rho_{+}-\rho_{-}$. While we know $\rho_{+}+\rho_{-}+\rho_{0}=1$, so the features of $\rho_{+}$ and $\rho_{-}$can be depicted entirely by $m$ and $\rho_{0}$. We define $\rho_{c}$ as the density of unsatisfied links, each of which links two nodes respectively with state +1 and -1 . We consider domain as a sub graph, and the homogeneous domains as the sub graphs of the graph only retaining the edges that link nodes with same states. And we take the relative size, namely scale of the largest one in these homogeneous domains into observation. We report all of above observables in the plane $(s, \varphi)$, and show them in a $3 \mathrm{D}$ curved surface mode in Fig.1.

\section{RESULTS AND EXPLANATION}

From the values of these observables showed in the Fig. 1 , we can find that there are three quite different phase areas which are showed in the Fig. 2. The area III is the most ordinary area, and the features on it are simple. It needs no more than 4 units to get frozen, the density of 0 is whole zero, and the density of +1 and -1 is equally 0.5 , and the density of unsatisfied links is also about 0.5 . There are several domains, and the largest of them keeps the size 0.5 . This holds much similarity with the initial situation, which means that there are not too many changes in this phase area.

In the phase II, it can still get frozen in several units, but the feature shows a great difference. The density of 0 doesn't change, but the magnetization comes to be either 1 or -1 . The density of unsatisfied links turns into 0 , while there is just 1 domain which occupies the whole graph. It means that in this situation, the whole society come into the same. The effects between people are sufficient and effective, and it is much like the situation in real events. But there is an uncertainty that whether +1 or -1 will be the stable condition, which seems to be determined by factors out of $s$ and $\varphi$. Between the phase areas II and III, there is a narrow but existing transition area $\mathrm{A}$. This area holds features like the phase area II, and is mingled with sparse features of the phase area III. But there is an evident increase in the convergence time, which we can comprehend as people's gradually convincing each other and being convinced by each other maybe a process of tanglement. 


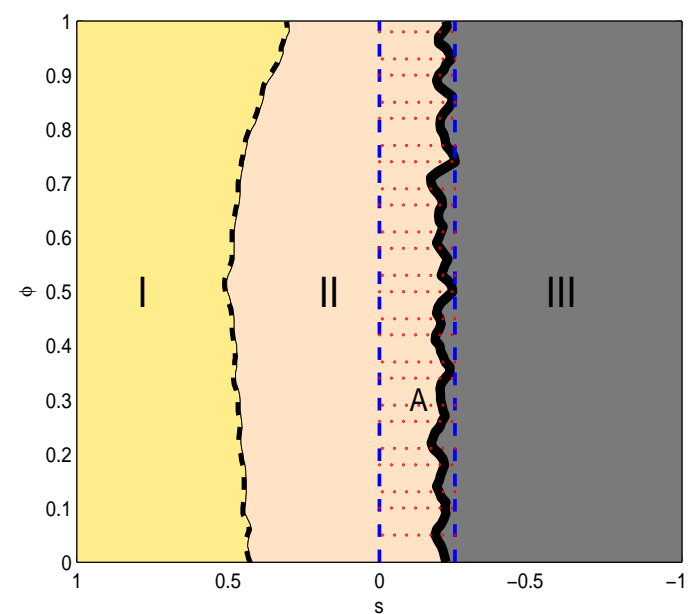

Figure 2. Schematic representation of the phase diagram obtained from numerical simulations on a graph with $N=10000,\langle k\rangle=10$, and the initial density of $0 \rho_{0}{ }^{\circ}=0$. Three phase areas can be obviously seen: the area I, II, and III, divided by the black lines, and there is a vague area A, which can be seen as the transition of phase areas II and III.

In the phase III, the evolution didn't end by itself. But the feature seems to be stable, and this situation could be seen as a combination of plenty evolution and endless recursion. In this area, the density of 0 changes suddenly to a no-small value, which we called critical phenomenon [14]. All of these observables, including the density of $0,+1,-1$, the density of unsatisfied links, the number of homogeneous domains, and the size of the largest domain etc., are mainly determined by $\varphi$. We show the information of domains when $s=1$ in the Fig. 3, and show that of density of 0 when $\mathrm{s}=1$ in the Fig. 4 .

In Fig. 3, it can be divided into 4 areas. The size of the largest domain decreases in the area (a) and (b), and then increase in the area (c) and (d). While the number of domains increases fast in area (a), it implements a gentle decrease in area (b). In the area (c), it increases again to even more than the most in the area (a) and (b). And in the area (d), it decreases a lot, and eventually goes to 1 . At the very beginning, there is a very small abstaining probability that the graph is occupied half by +1 and half by -1 ; the number of domains is 2 . Along with the increasing of abstaining probability $\varphi$, state 0 disarranges the original union of +1 and -1 . But it is still playing its role separately, until the area comes to (b), the state 0 gradually joins with each other. In the area (a) and (b), the largest domain is still composed of state +1 or -1 . While the area comes to (c), the situation changes a lot. State 0 processes the main part of the graph, and state $+1,-1$ are gradually separated into smaller and smaller domains. In the area (d), these separated states +1 or -1 begin to disappear, and the graph marches on to entire abstaining domain with a rapid speed.

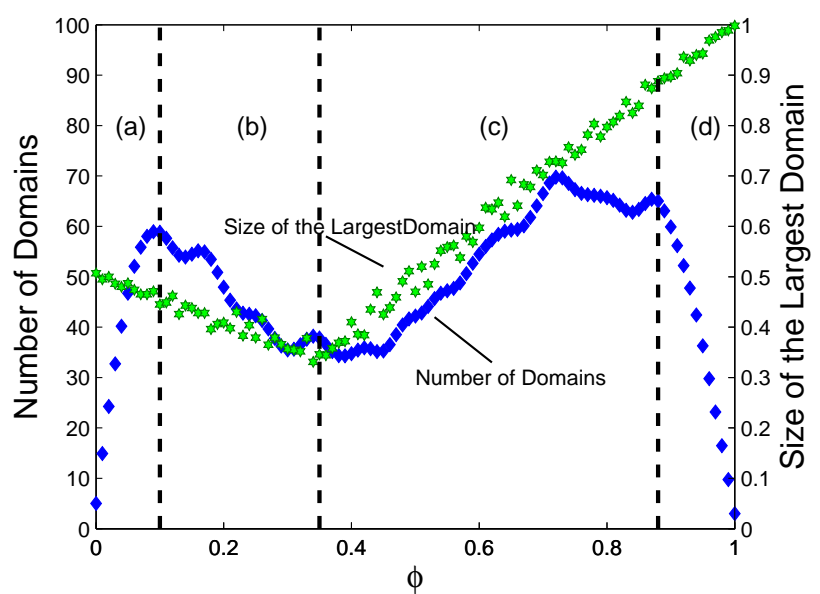

Figure 3. Number of domains and size of the largest domain as a function of $\varphi$ when $s=1$, obtained from numerical simulations on a graph with $N=10000,<k>=10$, and the initial density of $0 \rho_{0}{ }^{\circ}=0$.

From Fig. 1 and Fig. 4, we can figure out that the density of 0 increases linearly with the abstaining probability $\varphi$. It can be proved using mean field method originally from statistical physics. We have no much difficulty in finding that our model is the same with the following one, when $s>0$ :

(1) If $\left(n_{+}-n_{-}\right) / k>s$, the node adopts the state +1 ; If $\left(n_{+}-n_{-}\right.$ )$/ k<-s$, the node adopts the state -1 ;

(2) If $-s<\left(n_{+}-n_{-}\right) / k<s$, then take the following steps:

- with probability $\varphi$, the node adopts the state 0 (abstaining);

- with probability $1-\varphi$, the node adopts the state of +1 or -1 ; that is, if its original state is +1 or -1 , it stays the same, and if its original state is 0 , the node adopts the majority's state (intervention).

Then we have the following formulas,

$$
\left\{\begin{array}{l}
\frac{\mathrm{d} \rho_{+}}{\mathrm{d} t}=\sum_{\mathrm{k}=1}^{\infty} \frac{P_{k}}{1 / N} \sum_{\mathrm{ij}} B_{k, i, j}\left[\rho_{+} \theta_{1} \frac{-1}{N}+\rho_{-} \theta_{2} \frac{1}{N}+\rho_{0} \theta_{3} \frac{1}{N}\right], \\
\frac{\mathrm{d} \rho_{-}}{\mathrm{d} t}=\sum_{\mathrm{k}=1}^{\infty} \frac{P_{k}}{1 / N} \sum_{\mathrm{ij}} B_{k, i, j}\left[\rho_{+} \theta_{4} \frac{1}{N}+\rho_{-} \theta_{5} \frac{1}{N}+\rho_{0} \theta_{6} \frac{-1}{N}\right], \\
\frac{\mathrm{d} \rho_{0}}{\mathrm{~d} t}=\sum_{\mathrm{k}=1}^{\infty} \frac{P_{k}}{1 / N} \sum_{\mathrm{ij}} B_{k, i, j}\left[\rho_{+} \theta_{7} \frac{1}{N}+\rho_{-} \theta_{8} \frac{-1}{N}+\rho_{0} \theta_{9} \frac{1}{N}\right] .
\end{array}\right.
$$

In which, $P_{k}$ is the degree distribution, and $B_{k, i, j}=C_{k}^{i}$ $C_{k-i}^{j} \rho_{+}^{i} \rho_{-}^{j} \rho_{0}{ }^{k-i-j}$, the values of $\theta_{i}$ are as below:

TABLE I. THE VALUE OF $\Theta_{I}$ IN (1)

\begin{tabular}{c|c|c|c|c|c|c|c|c|c}
\hline condition & $\boldsymbol{\theta}_{\boldsymbol{1}}$ & $\boldsymbol{\theta}_{2}$ & $\boldsymbol{\theta}_{3}$ & $\boldsymbol{\theta}_{4}$ & $\boldsymbol{\theta}_{\boldsymbol{5}}$ & $\boldsymbol{\theta}_{\boldsymbol{6}}$ & $\boldsymbol{\theta}_{7}$ & $\boldsymbol{\theta}_{\boldsymbol{8}}$ & $\boldsymbol{\theta}_{\boldsymbol{9}}$ \\
\hline $\boldsymbol{( \boldsymbol { i } - \boldsymbol { j } ) / \boldsymbol { k } < - \boldsymbol { s }}$ & 1 & 0 & 0 & 0 & 0 & 1 & 1 & 0 & 1 \\
\hline $\boldsymbol{- \boldsymbol { s } < ( \boldsymbol { i } - \boldsymbol { j } ) / \boldsymbol { k } < \boldsymbol { s }}$ & $\varphi$ & 0 & $1-\varphi$ & $\varphi$ & $\varphi$ & $1-\varphi$ & 0 & $\varphi$ & $1-\varphi$ \\
\hline$(\boldsymbol{i}-\boldsymbol{j}) / \boldsymbol{k}>\boldsymbol{s}$ & 0 & 1 & 1 & 0 & 0 & 1 & 0 & 1 & 0 \\
\hline
\end{tabular}

When $s=1$, we have 


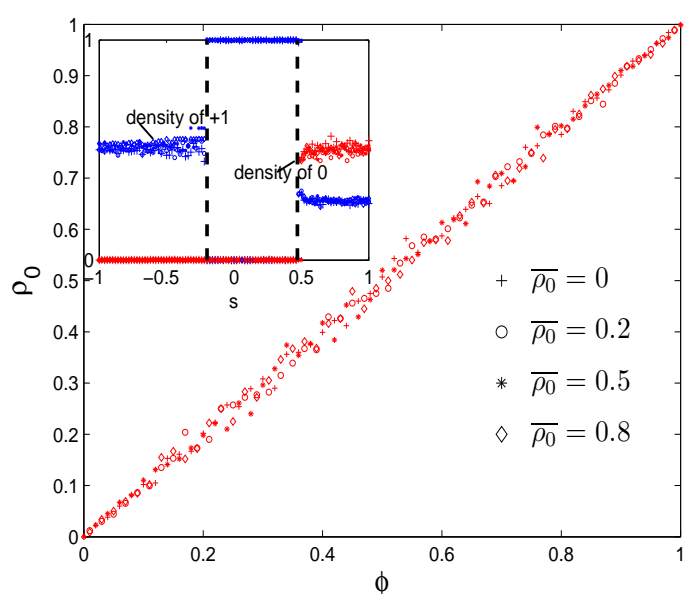

Figure4. (main) Density of 0 as a function of $\varphi$ when $s=1$; (sub) Density of 0 and +1 as a function of $s$, when $\varphi=0.5$; obtained from numerical simulations on a graph with $N=10000,<k>=10$, and the initial density of 0 $\rho_{0}{ }^{o}=0,0.2,0.5,0.8$.

$$
\left\{\begin{array}{l}
\frac{\mathrm{d} \rho_{+}}{\mathrm{d} t}=1 \cdot\left[\rho_{+}(-\varphi)+\rho_{0}(1-\varphi) \frac{1}{2}\right]=0 \\
\frac{\mathrm{d} \rho_{-}}{\mathrm{d} t}=1 \cdot\left[\rho_{-}(-\varphi)+\rho_{0}(1-\varphi) \frac{1}{2}\right]=0 .
\end{array}\right.
$$

Solve it with

$$
\rho_{+}+\rho_{-}+\rho_{0}=1
$$

We can obtain that $\rho_{+}=\rho_{-}=(1-\varphi) / 2, \rho_{0}=\varphi$. Now the linearity between the density of 0 and the abstaining probability has been proven.

We have confirmed from Fig. 1 that the state 0 has a phase transition from the phase area II to III. Through several experiments, we can also find that the emerging of abstaining does not vary with different initial densities of 0 . This can be easily seen in Fig. 4, both the main part and the sub part, that different $\rho_{0}{ }^{o}$ lead to the same trend.

\section{CONCLUSION AND FETURE WORK}

The model considering abstaining shows plentiful features in phase diagram. One of the most important results we get from this model is that the emerging of abstaining in a wide spread is determined not by the abstaining probability, but by the threshold which sets the limit to be stable. This proposition informs us a possible way to avoid the appearance of wide abstaining by controlling over the threshold. Other important outcome is that the observables stay the same in frozen state in all values of initial density of 0 . According to this, problem can be quite simplified.

The feature in the middle phase area attracts us a lot, but the factors that affect the graph to reach stable condition of either +1 or -1 are still unknown. The phase transition happens on the critical line, if the line can be possibly solved, the problem then gets a great progress.

\section{ACKNOWLEDGMENT}

This work was supported by the Natural Science Foundation of China (Grant No. 11201019).

\section{REFERENCES}

[1] Jr. French, R. P. John, "A formal theory of social power," Psychological Review, vol. 63, May 1956, pp.181-194, doi: 10.1037/h0046123.

[2] S. Mandra, S. Fortunato, and C. Castellano, "Coevolution of Glauberlike Ising dynamics and topology," Physical Review E, vol. 80, November 2009, doi: 10.1103/PhysRevE.80.056105.

[3] R. Hegselmann, U. Krause, "Opinion dynamics and bounded confidence models, analysis, and simulations," JASSS, vol. 5, no. 3, 2002.

[4] M. Granovetter, "Threshold models of collective behavior," Am. J. Sociol., vol. 83, no. 6, May 1978, pp.1420-1443.

[5] P.Clifford and A. Sudbury, "A model for spatial conflict," Biometrika, vol. 60, iss. 3, 1973, pp. 581-588, doi: 10.1093/biomet/60.3.581.

[6] R. A. Holley and T. M. Liggett, "Ergodic theorems for weakly interacting infinite systems and the voter model," The Annals of Probability, vol. 3, no. 4, August 1975, pp. 643-663.

[7] S. Redner, A Guide to First-passage Processes, Cambridge University Press, 2001.

[8] R. J. Glauber, "Time-dependent statistics of the Ising model," J. Math. Phys., vol. 4, iss. 2, February 1963.

[9] S. Galam, "Sociophysics: A review of Galam models," Int. J. Mod. Phys. C, vol. 19, iss. 3, March 2008, doi: 10.1142 /S0129183108012297

[10] K. Sznajd-Weron and J. Sznajd, "Opinion evolution in closed community," Int. J. Mod. Phys. C, vol. 11, iss. 6, September 2000, pp.1157-1165.

[11] K. Sznajd-Weron, "Dynamical model of Ising spins," Phys. Rev. E, vol. 70, iss. 3, September 2004, doi: 10.1103/PhysRevE.70.037104.

[12] P. Erdös and A. Rényi, "On random graphs," Publ. Math. Debrecen, vol. 6, 1959, pp. 290-297.

[13] P. Erdös, A. Rényi, “On the evolution of random graphs," Publ. Math. Inst. Hung. Acad. Sci., vol. 5, 1960, pp.17 60.

[14] J. J. Binney, N. J. Dowrick, A. J. Fisher and M. Newman, The Theory of Critical Phenomena, Oxford University Press, 1992. 